\title{
MEJORAS SOCIALES Y NEGOCIACIÓN COLECTIVA ${ }^{1}$
}

\author{
Ramón López Fuentes \\ Prof. Contratado Doctor, Departamento de Derecho del Trabajo y Seguridad Social, \\ Universidad de Sevilla
}

DOI: $10.1387 /$ lan-harremanak.15403

\section{ABSTRACT}

La regulación de la negociación colectiva, al igual que otros aspectos de la legislación laboral, ha sido objeto de diferentes reformas en los últimos años. Reformas que han tenido diferente repercusión a la hora de fijar posibles limites a la libertad negociar de las partes, tanto formales, como materiales. Argumentos que se han tendido a justificar en base a que asi era posible afrontar mejor la crisis económica existente. Argumentos que han servido de freno al desarrollo de las materias y contenidos propios de las denominadas "cláusulas sociales» e, incluso, a la desaparición de las mismas. Es cierto que no se trata de una tendencia general, pero si bastante generalizada. Sobre todo a nivel de convenios y acuerdos de empresa, amén de que la materia haya pasado a tener cada vez menor importancia en fórmulas de negociación supraempresarial. Tendencia en la que ahonda el hecho de acudir en el ámbito empresarial a fórmulas más flexibles y visualmente más positivas sobre el protagonismo de la empresa o grupo, como ocurre con las acciones propias de las empresas socialmente responsables.

Palabras clave: Negociación Colectiva. Contenido y Limites. Derecho de la Protección Social. Crisis económica. Responsabilidad Social.

${ }^{1}$ EURLAB SEJ-103. 
The collective bargaining regulation, as other aspects of labor law, has been under several reforms in the last years. The impact of these reforms has been different due to the fact that the limits to the negotiation freedom for both parts, have been affected, not only in a formal way but also in a material sense. This kind of arguments has tended to be justified as a mechanism to improve the way for affording the economic crisis. However, it also meant a slow-down in the development of what has been known as "clausulas Sociales" (Social Clauses), or even its disappearance. Although it is not a general tendency, it is true that it is even more extended, specially in the matter of collective agreements, without mentioning the subjects that had been released to a less relevant position in the business groups bargaining. This situation has been motivated companies to search new and a more positive corporate image. An example of this might be the socially responsible entrepreneurship.

Key Words: Collective bargaining. Contents and Limits. Social Protection rules. The economic crisis. Social responsability. 


\section{Introducción}

Nuestro trabajo no pretende ser un análisis clásico, en torno a qué consideremos cláusulas de protección social en la negociación colectiva; centrándonos en cómo definirlas y dónde ubicarlas — planteando si se catalogan como materias obligacionales o cómo contenido normativo- Para, a continuación, ver cómo ha evolucionado la materia en el contexto de crisis económica existente, teniendo presente el cambio de marco normativo que ha sufrido el papel de la negociación colectiva tras las sucesivas reformas operadas en la legislación laboral e incluso cerrando el tema en torno a la posible aplicación de esas premisas al contenido de los acuerdos profesionales de los TRADE. También podría incluirse alguna referencia a si es posible entender diversos niveles de aplicación en la materia, o en cuándo sería posible articular reglas de interpelación entre los diversos instrumentos para la negociación colectiva.

Podríamos enlazar tal cantidad de cuestiones, que difícilmente cumpliríamos nuestro objetivo inicial de señalar la realidad del tema en el espacio limitado al que hemos de ceñirnos. De ahí, que necesariamente nos limitemos a senalar ideas para un posible debate posterior. Por ello, nuestro punto de partida enlaza con el hecho de que el Derecho de la Protección Social extienda su alcance vía negociación colectiva: en cuanto fórmula-guía para desarrollar mejoras sociales en las personas destinatarias de su regulación. Para eso es necesario hacer un primer análisis positivo que vaya más allá de la tradicional aplicación de las mejoras voluntarias en materia de Seguridad Social, o de la llamada Acción Social de la Empresa en los Convenios Colectivos, a modo de primer texto de trabajo.

Entendido que solo así y, a partir de posteriores desarrollos, tendríamos una visión más amplia sobre el tema, donde cabe apostar por incluir una regulación innovadora que incluya y ampare cualesquiera otros aspectos que se nos ocurran para mejorar las condiciones de vida de los trabajadores y sus familias, por el hecho de desempeñar su actividad en un determinado entorno socioeconómico, que es objeto de regulación por diferentes instrumentos colectivos. 


\section{Marco normativo de referencia en la materia}

El art. 85 del TRET permite afirmar que el contenido de la negociación colectiva se caracteriza por su amplitud, en línea a dar así pleno contenido a los arts. 28 y 37 de la CE, e insertar nuestra regulación en consonancia con otras obligaciones internacionales ${ }^{2}$. Pues, dentro del respeto a las leyes, los convenios colectivos podrán regular materias de índole económica, laboral, sindical y, en general, cuantas otras afecten a las condiciones de empleo y al ámbito de relaciones de los trabajadores y sus organizaciones representativas con el empresario y las asociaciones empresariales.

Por tanto, el contenido del convenio queda al arbitrio y libertad que puedan acordar las partes firmantes, aunque con un triple límite de partida:

1. Que sean materias y aspectos relacionados con el ámbito sociolaboral. Y, por tanto, que regulen materias o cuestiones que puedan entroncar con la existencia de relaciones laborales entre las partes. Sin perjuicio, de que, a su vez, ese marco regulador pueda afectar a terceros, ya sea porque existan vínculos familiares o porque formen parte de posibles extensiones triangulares.

2. Que, en cualquier caso, dicho contenido respete la ley, tanto formal, como materialmente. Lo que supone, a su vez, indagar sobre el verdadero alcance de materias centrales en materia social.

3. $\mathrm{Y}$, sin que el contenido del cualquier acuerdo implique transversalmente tener presente el deber de negociar medidas dirigidas a promover la igualdad de trato y de oportunidades entre mujeres y hombres en el ámbito laboral o, en su caso, planes de igualdad; con el alcance y contenido previsto en el capítulo III del Título IV de la Ley Orgánica para la igualdad efectiva entre mujeres y hombres ${ }^{3}$.

Hechas las precisiones anteriores, parece perfectamente lógico señalar que en la negociación colectiva las partes pueden acordar lo que sus firmantes consideren oportuno, ya sea bajo la consideración de mejoras voluntarias o como previsiones que amplíen o regulen ex novo aspectos no contemplados en nuestro ordenamiento. El problema surge de forma inmediata ¿por qué no solo no se hace

\footnotetext{
2 Dado el peso aflictivo e interpretativo que hemos de dar a los convenios OIT ratificados por nuestro país, sino incluso de la propia Carta Social Europea; de los textos fundacionales de la UE o de la Carta de Derechos de la UE...vid.

3 Límite que hay que analizar ahora también a la luz del Acuerdo del Consejo de Ministros de 20 de noviembre de 2015, por el que se aprueba el II Plan para la Igualdad entre mujeres y hombres en la Administración General del Estado y en sus organismos públicos (BOE 10-12-2015), así como, en su caso, respecto al Protocolo de actuación para eliminar u limitar la violencia de género en el ámbito laboral.
} 
un mayor uso de dicha potestad, sino que incluso la misma ha visto reducido su campo de acción en numerosos casos? La respuesta, obviamente, no es unívoca y, aún a riesgo de no poder cerrar plenamente el tema, sí que admite algunas consideraciones al respecto.

\section{Límites estructurales al contenido de la negociación colectiva}

Pese a esa inicial idea de libertad de contenidos, proceden algunas precisiones adicionales, tanto desde un punto de vista formal, como material, para así poder centrar el debate, en cuanto que esas premisas añadan nuevas limitaciones para desarrollar contenidos en la materia o, por lo menos, nos aporten algunas respuestas adicionales al porqué se aplican o no determinados contenidos en la negociación colectiva.

Por un lado, porque el TRET establece mecanismos por los que dicha libertad de contenido puede verse condicionada en su aplicación por la estructura de la negociación colectiva con el nuevo marco referencial respecto a posibles reglas de concurrencia de convenios. Contenido que es especialmente patente en las regulaciones que establecen los Acuerdos Interprofesionales y Acuerdos Marcos - incluso los denominados Acuerdos Marcos Internacionales-, así como en relación a las relaciones entre convenios y acuerdos sectoriales —estatales o autonómicos-.

Ya que hemos de considerar las previsiones incluidas en los diferentes instrumentos de la negociación colectiva a la hora de articular relaciones con otras unidades negociables respecto a las diferentes materias objeto de regulación, y cómo ello afecte en la aplicación y alcance de las mismas. Y no solo porque se establezcan reglas en cuanto a establecer un cierto orden de prelación de la negociación colectiva, sino porque incluso se establezcan mecanismos de reservas o prioridades materiales entre convenios de diferentes ámbitos, y con diverso alcance:

a) Sea porque la materia se regula de forma muy general — para que desarrolle a posteriori por otro, $\mathrm{u}$ otros convenios-.

b) $\mathrm{O}$ porque se reserve materialmente el contenido de dicho convenio, y se limite la acción de cualquier otro de ámbito inferior.

c) Pero también puede ocurrir que se regule un bloque material de forma independiente, aunque el mismo, en su aplicación, a su vez se proponga de forma condicionada, por cuanto haya reservas o remisiones al respecto, etc.

En consecuencia, no creemos que podamos afirmar u optar por una regla unívoca a tener presente en la materia. Sino que, lo más probable, es que nos 
encontremos con una regulación interconectada o en «escalera» entre los diferentes instrumentos normativos que han de interrelacionarse entre sí para dar un sentido completo a la materia.

De cualquier forma, lo que sí deja claro nuestro legislador, —amén de los límites indicados en el apartado anterior-, es que dichos acuerdos no puedan disponer de la prioridad en su aplicación de determinadas materias presentes en un determinado ámbito. Así ocurre respecto a la inaplicación y reserva material que se hace en favor de los acuerdos a nivel de empresa del propio art. 83.2 del TRET —en cuanto que no cabe corregir la posibilidad de descuelgue de condiciones-, ni tampoco cabe limitar la reserva material que se establece en favor de los convenios de empresa en determinadas materias.

Siendo así que, y en segundo término, todo lo anterior siempre puede ser disponible a nivel de empresa, al menos, inicialmente ${ }^{4}$ :

a) Pues se establece una reserva material de carácter preferente al contenido de ciertas materias y condiciones, y respecto a lo que puedan regularse en otros convenios de aplicación. Dicha preferencia lo es respecto del contenido de un convenio sectorial estatal, autonómico o de ámbito inferior en una serie de materias donde, a lo que nos interesa, destaca la afección de las previsiones encaminadas a favorecer la conciliación entre la vida laboral, familiar y personal. Todo ello, sin perjuicio de todas aquellas otras que dispongan los acuerdos y convenios colectivos a los que se refiere el artículo 83.2 del TRET, como ya hemos visto.

b) Y la posibilidad, justificándolo por el carácter sobrevenido de unas determinadas circunstancias, de aplicar mecanismos de descuelgue de condiciones sociolaborales reconocidas en otros convenios y acuerdos previos — sean o no supraempresariales_-, cuando concurran causas económicas, técnicas, organizativas o de producción ${ }^{5}$. Excepción esta que obliga a cumplir determinados requisitos formales ${ }^{6}$ a nivel de los

${ }^{4} \mathrm{Y}$ decimos «inicialmente» por cuanto podemos anticipar ya que pueden existir diferentes aspectos que condicionen dicha materia. Ya sea desde un plano fiscal, en relación con la Seguridad Social, con el tamańo de las empresas, con el papel de la Responsabilidad Social, etc., que pueden repercutir en un mayor o menor alcance de las medidas a considerar.

5 Se entiende que concurren causas económicas cuando de los resultados de la empresa se desprenda una situación económica negativa, en casos tales como la existencia de pérdidas actuales o previstas, o la disminución persistente de su nivel de ingresos ordinarios o ventas. En todo caso, se entenderá que la disminución es persistente si durante dos trimestres consecutivos el nivel de ingresos ordinarios o ventas de cada trimestre es inferior al registrado en el mismo trimestre del año anterior. Es más, pese a todo lo anterior, y siempre y cuando concurran determinadas causas, es posible incluso la inaplicación de las citadas cláusulas cuando concurran las circunstancias indicadas en el art. 82 del TRET.

6 Y siempre que medie acuerdo entre la empresa y los representantes de los trabajadores legitimados para negociar un convenio colectivo conforme a lo previsto en el artículo 87.1 del TRET. 
acuerdos y convenios de empresa ${ }^{7}$, pero también respecto a los contenidos. Y que a lo que nos interesa, se centraría en aquellas materias que afecten a mejoras voluntarias de la acción protectora de la Seguridad Social, pero no tendría por qué ser aplicable a otros ámbitos materiales relacionados con la materia.

Tanto en un caso, como en el otro, sí hay que señalar que la posibilidad de negociar estará abierta en cualquier momento de la vigencia de otros convenios colectivos, o incluso a falta de los mismos.

\section{Límites materiales sobre qué considerar como contenido social de la negociación colectiva}

Siendo importante justificar cómo y dónde se ha regular la materia social del convenio, sin duda lo primero es circunscribir qué se entiende como contenido social del Convenio. Esto es qué puede tener cabida en el convenio respecto a esta materia.

La respuesta, a nuestro juicio, y como venimos indicando ha de ser amplia $^{8}$. Pues, y sin perjuicio de establecer un claro nexo constructivo entre el Derecho de la Protección Social y el de Seguridad Social, hay diferencias de extensión. Al igual que ocurre cuando diferenciamos el género y su especie, siendo el derecho de la Protección Social el primero, e iría más allá de la Seguridad Social. Luego, las medidas sociales de la negociación colectiva, pueden integrarse en ese concepto omnicomprensivo del Derecho a la Protección Social, en cuanto instrumento adicional y necesario para su desarrollo en el ámbito de lo privado.

Partiendo de esa premisa, la Negociación Colectiva quedaría como instrumento abierto para el desarrollo de los denominados derechos fundamentales de los trabajadores ${ }^{9}$, pudiendo dar cabida a cualquier materia de índole social y, por definición, de carácter abierto a todos los temas que van más allá de las estrictas condiciones de trabajo, pero que pueden derivarse del complejo ámbito en el que se desarrollan aquellas. Completando así un cuadro de derechos y obligaciones variopinto, cuyo hilo conductor radica en la libertad de las pa-

7 Igual prioridad de aplicación tendrán en estas materias los convenios colectivos para un grupo de empresas o una pluralidad de empresas vinculadas por razones organizativas o productivas y nominativamente identificadas a que se refiere el artículo 87.1.

8 En cuanto que la materia entroncaría con el denominado Derecho de la Protección Social y aquel se caracteriza por su amplitud.

9 Tal y como reconocen diferentes normas Internacionales del trabajo, como la Carta Social Europea y el TUE y, sobre todo, por las Naciones Unidas. 
tas para mejorar las condiciones sociolaborales, tanto en el plano individual, como colectivo.

Pues, no olvidemos que el trabajo no es solo un instrumento para la obtención de rentas, "...sino un factor de desarrollo personal y social, que bien merece una atención integral en cuanto instrumento para la promoción del bienestar humano y el consenso social, que favorece la paz social y es indispensable para lograrla, y por lo tanto para mejorar el crecimiento equitativo, la estabilidad social y el desempeño económico, contribuyendo a la competitividad», como la propia OIT establece ${ }^{10}$ y que, por tanto, todo aquellos que tiene que ver con su marco normativo debe estar condicionado por esa concepción garante de derechos.

Sin embargo, y pese a esa amplitud conceptual, al analizar diferentes convenios $\mathrm{y}$ acuerdos ${ }^{11}$, apreciaremos dos ideas casi reiterativas en los últimos años.

a) Las pocas novedades que a la materia se aportan. Esto es, una negociación colectiva muy pegada a directrices generales a nivel de convenios supraempresariales. Muy limitada a reconocer temas en torno a la mejora de algunas prestaciones de Seguridad Social y de la denominada Acción Social en la empresa, y donde sin duda podría trabajarse más en materias tales como el empleo —en cuanto instrumento de inserción de determinados colectivos—, de conciliación, de desarrollo profesional,

10 Por lo que, y sin llegar a un planteamiento tan amplio en cuanto al contenido que la negociación colectiva ha de cumplir en relación a la Protección Social, sí consideramos necesario postular por su papel instrumental y complementario a las políticas sociales y de empleo que desde las AAPP puedan establecerse, en cuanto fin último para considerar estándares en torno al "trabajo decente» y consecución de Pisos de Protección Social. Al respecto vid. www.ilo.com, Protección Social, en cuanto consideración de que la Protección Social es uno de los cuatro objetivos estratégicos del Programa Trabajo Decente, que contiene principios fundamentales para el trabajo de la OIT. Desde su creación en 1919, la OIT promueve políticas y ofrece a los Estados miembros instrumentos y asistencia con el objetivo de mejorar y extender la cobertura de la protección social a todos los grupos sociales y a mejorar las condiciones y la seguridad laboral. De forma que la OIT, en materia de protección social, ha establecido tres objetivos principales que reflejan las tres dimensiones fundamentales de la protección social:

1. Aumentar la eficacia y extender la cobertura de los sistemas de seguridad social

2. Promover la protección de los trabajadores, que incluye: condiciones de trabajo decentes, como salarios, tiempo de trabajo y salud y seguridad en el trabajo, todos componentes esenciales del trabajo decente

3. Promover la protección de grupos vulnerables, como los trabajadores migrantes, sus familias y los trabajadores en la economía informal, mediante programas y actividades específicos. Además, se utilizará todo el potencial del mundo del trabajo para responder a la pandemia del SIDA, con especial atención al fortalecimiento de la capacidad de los mandantes tripartitos

${ }^{11} \mathrm{Al}$ respecto vid. referencia web CNCCC y CARL. 
cultura y formación, o vivienda.... Y ello, sólo por poner ejemplos que sin llegar a identificar con todos los aspectos del Derecho de la Protección Social, sí que permitan establecer parámetros de referencia que, al menos desde la redacción del propio art. 85 TRET sean posiblemente más amplios que las actuales regulaciones en torno a las mejoras voluntarias en materia de Seguridad Social ${ }^{12}$, completando también la denominada Acción Social, las materias relacionadas con la Discapacidad, o el Género.

b) Una tendencia, generalizada, que no general ${ }^{13}$, a que esas mismas cláusulas convencionales vistas en el apartado a) se hayan visto afectadas por la actual situación económica. Minorando, en la mayor parte de los casos, su campo de acción. $\mathrm{O}$ mejor dicho, con la "coletilla» de que bajo el paraguas de la situación económica negativa, el contenido social de la negociación colectiva ha visto reducido su posible aplicación. Bien porque deja de ser aplicable la negociación colectiva previa; porque hay un descuelgue de condiciones. E incluso por el desarrollo de decisiones legislativas que podemos encuadrar en el ámbito recaudatorio ${ }^{14}$, o de desarrollo estructural en determinados colectivos ${ }^{15}$... Aunque también hay que resaltar cómo la situación económica ha permitido un cierto desarrollo empresarial de la materia en ciertos sectores y ámbitos profesionales, si bien, huyendo del ámbito de la negociación colectiva. Eso es lo que ha ocurrido con la inclusión de medidas sociales para sus trabajadores dentro de las acciones derivadas de la adopción de criterios de Responsabilidad Social corporativa o de Gestión Socialmente Responsable de ciertas Empresas y Grupos de Empresas, así como a través de medidas sociales vinculadas a Fundaciones y Entidades no Gubernamentales apoyadas empresarialmente ${ }^{16}$.

12 Por más que algunos de ellos, y como hemos señalado por el juego de las previsiones sobre articulación de la negociación colectiva y por la reserva material y posible inaplicación a nivel de empresa, puedan quedar supeditados a decisiones económicas posteriores y, por tanto, en entredicho su posible desarrollo y aplicación

13 Por cuanto, y pese a lo que pudiese pensarse, no todos los sectores, ni empresas han ido hacia atrás en la materia, si no que hay algunos ejemplos realmente interesantes, y no sólo en el ámbito de las nuevas tecnologías.

${ }^{14}$ Como se ve de un análisis en profundidad de las medidas fiscales y de cotización que se han ido adoptado en los últimos ejercicios. En línea a establecer una equiparación en el tratamiento de determinadas compensaciones y mejoras, equiparándolas a verdaderas rentas del trabajo dichas mejoras.

15 Como ha ocurrido respecto a la negociación colectiva en el sector público en cuanto normativamente se ha ido atacando a un importante «colchón» de medidas sociales de dichos colectivos

16 Ya que a la empresa le supone "poco cambio efectivo", pero directamente gestiona la materia, amén de poder excluir, en su caso, a los propios representantes de los trabajadores, a la vez que incluso puede dar cabida a otros colectivos que estarían al margen de posibles pactos, acuerdos o convenios. Teniendo, además, un cierto efecto llamada o de publicidad positiva al respecto. 


\section{Conclusiones}

A la vista de todo lo anterior, podemos afirmar que la libertad inicialmente reconocida para fijar contenidos sobre mejoras sociales está condicionada por límites estructurales de la negociación colectiva, conforme a las previsiones del art. 85 del TRET ${ }^{17}$. En concreto, nos referimos a aquellos casos en los que se establece la posibilidad de dejar reservadas o fijar preferencias en la aplicación de determinadas materias entre convenios de diversos ámbitos. Sean bloques materiales concretos. $\mathrm{O}$ sea en cuanto al cómo han de integrarse determinados aspectos entre sí - como si de "campos acotados de aplicación preferente» se tratase- ${ }^{18}$.

Por tanto, la reglas introducidas por las reformas laborales sobre la relación entre convenios de diferente ámbito no solo puede condicionar qué se aplica, si no cómo se aplica dicho contenido. Por lo que si nada parece impedir que los convenios y acuerdos colectivos contengan cláusulas relacionadas con la Protección Social de los trabajadores, tampoco ello puede ser entendido en plena libertad a la hora de su establecimiento, si no que estará sometido a un complejo sistema de interpelación normativa, así como al propio deseo de las partes firmantes.

Luego, no solo hay límites estructurales; sino de voluntad negociadora. Lo que nos ha de llevar a un debate sobre qué entendamos por contenido social de la negociación colectiva, y su relación con el Derecho de la Protección Social. Punto de partida que obliga a considerar varios factores de orden metodológico, donde no creemos que proceda establecer una lista cerrada de materias, salvo cuando se trate de materias imperativas que, en cualquier caso, también pueden ser completadas. Complementando así al sistema ya existente a la hora de mejorar las condiciones de vida de dichos trabajadores, de sus familiares y hasta de otros posibles terceros que puedan verse afectados por esas relaciones sociolaborales.

17 Que, a su vez, pueden buscar su cambio o inaplicación a partir de circunstancias económicas o económicamente evaluables. Si bien, en estos casos, con un cierto alcance limitado, al menos formalmente. Pues, en sentido práctico, ya veremos que si hay algo renunciable y renegociable son las cláusulas «de contenido social»: en función del ámbito de negociación, y de la realidad económica en la que se desarrolle el proceso de negociación.

18 Ejemplo de lo anterior es lo que ocurre cuando se establece la posible inaplicación de condiciones más allá de las propias reservas que el art. 83 del TRET establece. Pues no olvidemos que en caso de posible concurrencia entre convenios, hay materias que si están reguladas a nivel de empresa gozan de criterio preferencial en su aplicación, no siendo dichas materias objeto de posible renuncia en cuanto a las reglas de concurrencia. Amén de que el descuelgue de determinadas condiciones proceda en cualquier caso que así se justifique por razones de índole económica, como establece el propio TRET. 